\title{
A Social Network-Based Architecture for On-Line RoSCAs in the Developing World
}

\author{
Gilles Kounou $^{\# 1}$, Christian Akpona ${ }^{\# 1}$, Herve Ahouantchede ${ }^{\# 1}$, Rose Gohoue ${ }^{\# 1}$, Fatna Belqasmi ${ }^{* 2}$, Roch \\ Glitho ${ }^{\# 1, \& 3}$ \\ ${ }^{\# 1}$ IMSP, Porto Novo, University of Abomey-Calavi, Republic of Benin \\ ${ }^{* 2}$ Zayed University, Abu Dhabi, United Arab Emirates \\ ${ }^{\& 3}$ Concordia University Montreal, Canada (glitho@ece.concordia.ca)
}

\begin{abstract}
Rotating Saving and Credit Associations (RoSCA) (known as tontines in francophone Africa) are financial systems involving groups of individuals who put money together periodically and take turns to collect the kitty. They are off-line systems and are very popular in the developing world. However, they now face several challenges (e.g. geographical distance between participants, safety risks related to manual financial operations). An evolution towards on-line systems is now a necessity and this paper is a first step in that direction. It proposes an architecture which handles the participants of ROSCAs as end-users of a social network. The proposed social network accommodates poorly literate end-users who may live in areas with no Internet connection. It also integrates an on-line payment system. The rich cellular network connectivity that is commonplace in most developing countries is used to offset the poor Internet connectivity. Speech technologies (e.g. Interactive Voice Response - IVR, speech to text - STT, text to speech - TTS) are used to address the literacy issue
\end{abstract}

\section{Categories and Subject Descriptors}

D.3.3 H3.4 Social Networks

\section{General Terms}

Design, Experimentation

\section{Keywords}

RoSCAs, tontine, social networks, voice enabled social network, poor Internet access

\section{INTRODUCTION}

Rotating Saving and Credit Associations (RoSCA), known by different names in different countries (e.g. osusu in Ghana, esusu in Nigeria, tontines in francophone Africa, bisi in Pakistan) are groups of individuals who agree to borrow and save together for a given period of time [1]. They are near ubiquitous in many parts of the developing world, especially in West Africa, where membership can run as high as 50 to $95 \%$ of the adult population in countries such as Nigeria and Cameroon [2].

Permission to make digital or hard copies of all or part of this work for personal or classroom use is granted without fee provided that copies are not made or distributed for profit or commercial advantage and that copies bear this notice and the full citation on the first page. To copy otherwise, or republish, to post on servers or to redistribute to lists, requires prior specific permission and/or a fee. ACM DEV-4, Dec 06-07 2013, Cape Town, South Africa ACM 978-1-4503-2558-5/13/12
RoSCAs are traditionally made up of individuals with strong off-line social connections, who live in close geographical proximity and have very rich face to face social interactions (e.g. group meetings) during the given period. The literacy level of the individuals is usually rather uneven and most (if not all) financial operations are carried out manually. Unfortunately, the fast pace of social changes makes this modus operandi less and less sustainable. We envision an online future for RoSCAs, with participants scattered geographically, interacting online most of the time and carrying out all their financial operations electronically. The main roadblocks to the realization of this vision are the poor literacy levels and the poor Internet coverage.

Mobile payment-based solutions, such as MTN Money [3] deployed in several African countries, can somehow aid in tackling the problem of manual financial operations. However, they do not address the issue of online social interactions, a problem that we believe on-line social networks can solve. Reference [4] proposes a social network for areas with poor Internet coverage, but it does not tackle the poor literacy issue. Reference [5] proposes a general architecture that enables poorly literate end-users to browse the Web. Unfortunately, it does not enable on-line interactions between end-users.

\section{PROPOSED ARCHITECTURE}

We use on-line social networks as the key enablers of our proposed architecture. Figure 1 depicts the main components.

Users can access our proposed on-line RoSCA via the cellular network which is now nearly ubiquitous in the developing world. SMS or voice can be used as the access mode. Having the option of voice as an access mode enables us to address the needs of poorly literate endusers; they can interact with the system via an interactive voice response (IVR) system, using vernacular languages. We should note that a few voice-enabled social networks have been proposed in the literature (e.g. [5]), but they assume Internet connectivity. The access layer interacts with the processing layer via a text-based interface. Speech to text (STT) and text to speech (TTS) technologies are used to bridge the two layers of the voice access module. The key module of the processing layer is the MobileTontineRequestHandler. It dispatches the request to either the CardChecking and MembershipManagementModule (on-line financial operations) or the SNRequestHandler, (social interactions operations). The mediation layer ensures that the social network is built on top of the existing social network infrastructure whenever possible. The standard Opensocial [6] is used for that purpose, making deployment possible on top of any existing social network which supports OpenSocial [6]. All of our architecture's interfaces rely on RESTful Wed services technology [7] to enable easy interoperability. 




Figure 1: Proposed architecture

\section{PROTOTYPE}

Figure 2 shows our proof of concept prototype.



SMSs and voice calls are routed to a SIM card on a GSM modem (i.e. HUAWEI E153). The SMSs are then transferred to the SMS access manager and the voice calls are transferred to the voice access manager. The IVR module is implemented using Asterisk [8], a free open-source software framework for building communications systems. The session initiation protocol (SIP) is used as the signaling protocol. Julius [9] is used for STT and Festival [10] for TTS. We customized them for FON, a vernacular language of the Republic of Benin. In the scenario, poorly iterated end-users pay online using FON by dictating the digits on the payment cards. We assume they are literate enough to read digits. We use an Oktell SIP-GSM gateway [11] to bridge between the GSM and SIP domains on both the signaling and the media planes. The back-end social network is implemented using Apache Shindig, the reference implementation of OpenSocial API specifications. Apache Shindig is open source and it assists in the building and hosting of social networks OpenSocial.

\section{CONCLUSIONS}

This paper proposes an online system for RoSCAs in the developing world. The system relies on social networks as enablers. A prototype has been built using tools such as Asterisk, Julius, Festival and Apache Shendig. In future work we plan to deploy the system on real social networks that support OpenSocial (e.g. Google+). We also plan to define ontologies for RoSCAs in order to enhance/improve the STT and TTS aspects of our work.

\section{REFERENCES}

[1] O. Anku-Tsede, Susu: A Dynamic Microfinance Phenomenon in Ghana, Journal of Economics and Sustainable Development www.iiste.org, ISSN 2222-1700 (Paper) ISSN 2222-2855 (Online)Vol.4, No.3, 2013, Page 146 - 155

[2] B. Agegnehu, Why do members join indigenous informal financial institutions - RoSCAs, Masters thesis No 710 - ISSN 1401-4084, Uppsala 2012

[3] U. Varshney, Mobile Payments, IEEE Computer, Volume 35, Issue:12, December 2002, , Pages 120 - 121.

[4] N. Vallina-Rodriguez,P Hui, J Crowcroft, "Has anyone seen my goose? Social Network Services in DevelopingRegions", 2009 International Conference on Computational Science and Engineering.

[5] S. Smaldone et al, RoadSpeak: Enabling Voice Chat on Roadways Using Vehicular Social Networks, SocialNets'08, April 2008.

[6] M. Hasel, Opensocial: An Enabler for Social Applications on the Web, Communications of the ACM, Volume 54 Issue 1, January 2011, Pages 139-144.

[7] F. Belqasmi, C. Fu, R. Glitho, RESTful Web Services for Service Provisioning in Next Generation Networks: A Survey, IEEE Communications Magazine, Vol. 49, No12, , December 2011, Pages 66-73.

[8] http://www.asterisk.org/.

[9] A Lee et al., Recent Development of Open Source Speech Recognition Engine Julius, APISIPA ASC 2009

[10] M. Beutnagel et al., The AT\&T next-gen TTS system, Joint Meeting of ASA, 1999.

[11] http://www.oktellpbx.com/gateways/, June 2013. 